\title{
Intracellular competition for fates in the immune system
}

\author{
Ken R. Duffy ${ }^{1}$ and Philip D. Hodgkin ${ }^{2,3}$ \\ ${ }^{1}$ Hamilton Institute, National University of Ireland, Maynooth, Ireland \\ ${ }^{2}$ Walter \& Eliza Hall Institute of Medical Research, Parkville, Melbourne, Australia \\ ${ }^{3}$ Department of Medical Biology, University of Melbourne, Melbourne, Australia
}

\begin{abstract}
During an adaptive immune response, lymphocytes proliferate for five to $\mathbf{2 0}$ generations, differentiating to take on effector functions, before cessation and cell death become dominant. Recent experimental methodologies enable direct observation of individual lymphocytes and the times at which they adopt fates. Data from these experiments reveal diversity in fate selection, heterogeneity and involved correlation structures in times to fate, as well as considerable familial correlations. Despite the significant complexity, these data are consistent with the simple hypothesis that each cell possesses autonomous processes, subject to temporal competition, leading to each fate. This article addresses the evidence for this hypothesis, its hallmarks, and, should it be an appropriate description of a cell system, its ramifications for manipulation.
\end{abstract}

Heterogeneity in the adaptive immune response The study of the regulation of cell death, division and differentiation is common to many biological systems. Understanding the control of these, often simultaneous, events and expressing them in quantitative terms is a key challenge for cell biology. The adaptive immune response offers an excellent model system because a small number of similar quiescent naïve lymphocytes ( $\mathrm{T}$ cells and/or B cells) are stimulated to undergo numerous rounds of division, thereby exponentially expanding the number of cells specific to the antigen. In parallel to this expansion, cellular differentiation produces heterogeneous populations of effector cells to meet the challenge of the threat posed by the pathogen [1-5]. Following the response many of the newly generated cells die by apoptosis, leaving a small number of long-lived effector and memory cells that provide rapid protection against repeated infection by the same pathogen $[2,6-12]$.

A typical B cell response is illustrated (Figure 1 illustrates the canonical pattern of an adaptive immune response and the broad heterogeneity of cell types that arise) during which a proportion of cells undergo an isotype switch in antibody type (IgM to IgG) [13], and both switched and unswitched cells develop into dividing plasmablast antibody-secreting cells (ASC), sessile antibodysecreting plasma cells and long-lived circulating memory cells. A similar pattern of response accompanied by the generation of different cellular types is observed for CD4

Corresponding author: Hodgkin, P.D. (hodgkin@wehi.edu.au).

Keywords: adaptive immune response; stochastic fate determination; autonomous competition hypothesis. and CD8 lymphocytes [14,15]. As a model for studying the regulation of cell proliferation, differentiation and cessation, the adaptive immune response has many desirable features: the response takes course over a period of two weeks; its key features can be recreated in vitro; there are well-understood cell-surface markers and genetically engineered reporter mice that enable identification of cell differentiation; and last, but not least, the response can be manipulated through the addition of cytokines that quantitatively alter many of its facets [16].

Since the 1980s substantial amounts of quantitative information on lymphocyte population dynamics has been obtained. One significant feature that has been revealed is that the response is consistent and repeatable for given stimulation conditions, both in vitro and in vivo. Another is that naive lymphocytes are multipotent, and single precursors can generate and populate a broad range of mature effector and memory cell types [17-19], raising the question of how consistent proportions of heterogeneous cell fates are achieved.

In answering this question, time-series data provided by flow cytometry are limiting because only indirect inferences can be drawn regarding fate determination of individual lymphocytes. To overcome this shortcoming, in recent years experimental methodologies that incorporate time-lapse imaging platforms have been developed to enable direct observations at the level of individual cells [20-28]. The emerging insights and open questions from the analysis of these experiments are the subject of the present article.

We expound upon, in particular, the autonomous competition hypothesis (ACH) [29-33]: that, to a first level of approximation, for each fate within a cell an autonomous process is initiated at birth or by an activating trigger that determines when that cell will possess that fate. The times to completion of these processes are ascribed probabilistic distributions and the observed fate of a cell is determined by competition of the completion times of these processes. A system that is well modeled by the ACH inexorably leads to the existence of nontrivial, intricate correlation structures in observed times to cell fates. These serve as the hallmarks of the hypothesis and are teased out here because their appearance or absence in data serve as essential tests of the appropriateness of the $\mathrm{ACH}$.

\section{Fate determination}

How does the observed heterogeneity in cell fate arise during an adaptive immune response? There is no doubt 


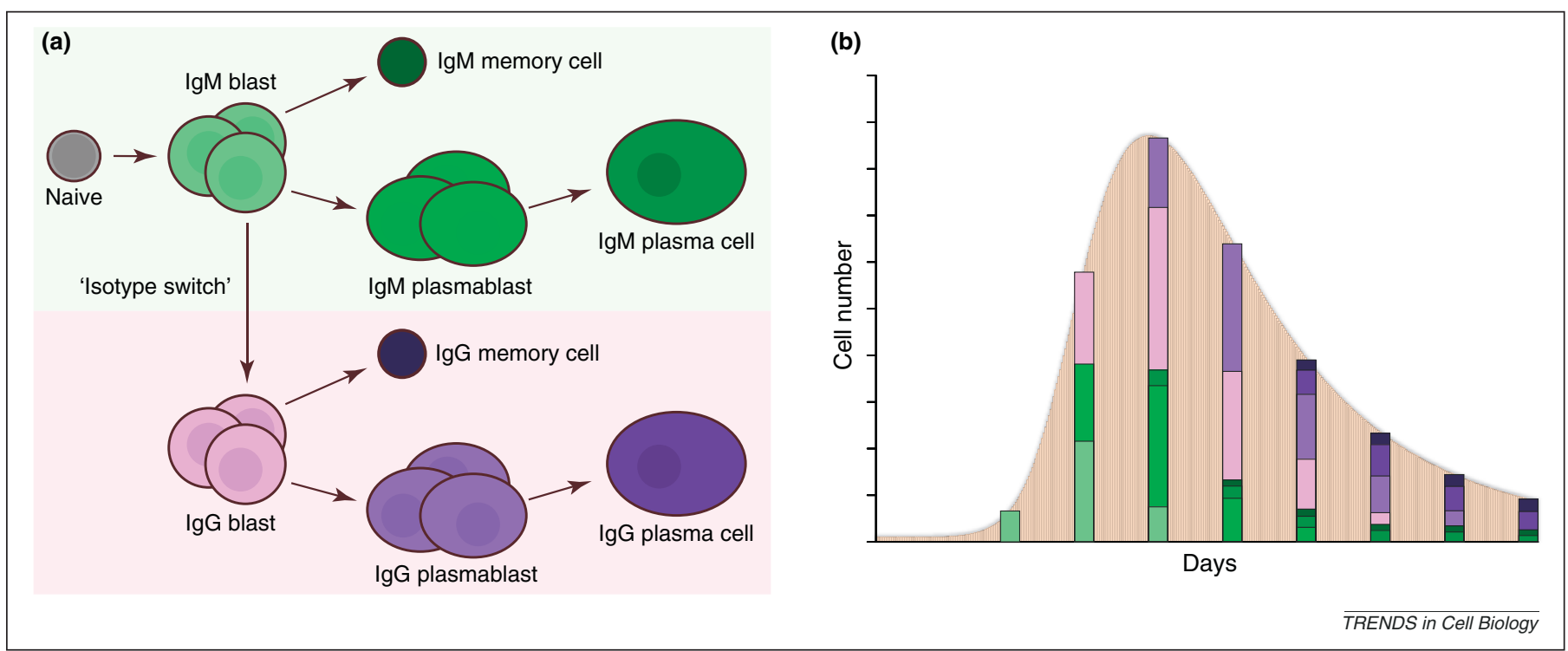

Figure 1. Canonical pattern of the adaptive immune response. (a) When stimulated by exposure to combinations of antigen and regulatory signals, lymphocytes begin a series of rapid divisions accompanied by differentiation to generate a large number of distinct effector and memory cell types. A small set of the prominent B cell subsets are shown in (a). Panel (b) illustrates the canonical pattern of proliferation, differentiation, cessation, and death in response to stimulus. After 5-20 generation rounds most cells die by apoptosis, leaving a small proportion of long-lived cells, either memory or antibody-secreting plasma cells, that provide ongoing protection. The timedependent emergence and loss of distinct cell types during expansion is shown by the colors matched to subsets illustrated in (a). T cells also have a vast range of effector and memory states and follow a similar canonical pattern of response to that shown in (b).

that lymphocytes can be regulated by cell-cell interactions, secreted cytokines, and external signals in general, a broad range of which have been identified - including several that govern cell migration. Even when many of these complicating factors are removed, for example by the addition of saturating concentrations of cytokines in vitro, similar cells give rise to highly varied outcomes [34-36]. This implies that mechanisms intrinsic to the cell play a significant role. It is this intrinsic heterogeneity, even in the absence of heterogeneous external signaling, that is our focus here.

During an adaptive immune response an important hidden variable in decision-making by a cell is the number of divisions (generation number) after the initial stimulus that gave rise to it. Studies using division-tracking dyes have repeatedly demonstrated that fate determination is tightly intertwined with generation number. Examples include the frequencies with which $\mathrm{B}$ and $\mathrm{T}$ cells take on effector states and the likelihood that they divide or die [37-44]. Significantly, the distributions of times from an activating trigger for a cell (or from its birth) at which various fate decisions take place also change as a function of generation $[22,33]$.

The heterogeneity in times to fate argues against a simple deterministic viewpoint where two progenitor cells exposed to the same stimulus will generate the same family tree, with asymmetric division at key points in the development of the tree giving rise to the exactly correct proportion of cells that are appropriate for the response. Instead, the heterogeneity is suggestive of a role for randomness in fate determination (Figure 2 for a list of potential sources) with population-level repeatability then arising as a consequence of laws of large numbers.

The simplest model incorporating randomness would be to suggest that each newly born cell makes its decisions de novo, independently of other cells. This is the starting point for most stochastic models [30,45-47]. Recent data, however, paint a more complex picture, one that reveals substantial heterogeneity across the family trees of distinct progenitors, but strong familial correlations within them [22,33].

These features of simultaneous regulation of multiple fates, including division, death, and differentiation, suggest a complex internal decision-making process within the cell. Despite the daunting nature of this system, a succinct, biologically reasonable paradigm is possible within which the apparent complexities are readily explained.

\section{The ACH}

The intracellular $\mathrm{ACH}$

For single cells, the ACH postulates that, from the birth of a cell or an activating trigger, distinct processes are underway leading to each individual fate. Each process can be described by a distribution of times from birth until the process to create that fate is completed. At a first level of approximation these processes are autonomous and the times are therefore modeled by independent random variables. Crucially, these processes are in competition and, consequently, particular values are censored. For example, if the division process completes before the death process, then the cell divides and the time at which the death process would have completed is censored and goes unobserved. From a mathematical modeling perspective, the origins of the randomness within cellular processes that underlies the ACH need not be known to explain single-cell behavior or to extrapolate to the population level, and this is a virtue of the hypothesis. By contrast, for cell biologists, the cellular and molecular processes that underpin this heterogeneity will be of primary interest. Here, we expand upon the recent evidence for the $\mathrm{ACH}$, aiming to attract further experimentation, hypothesis and dissection of the underlying molecular mechanisms by the cell biology community.

Before recent time-lapse microscopy experiments, evidence in support of the three main features of the intracellular ACH for lymphocytes - heterogeneity, independence 


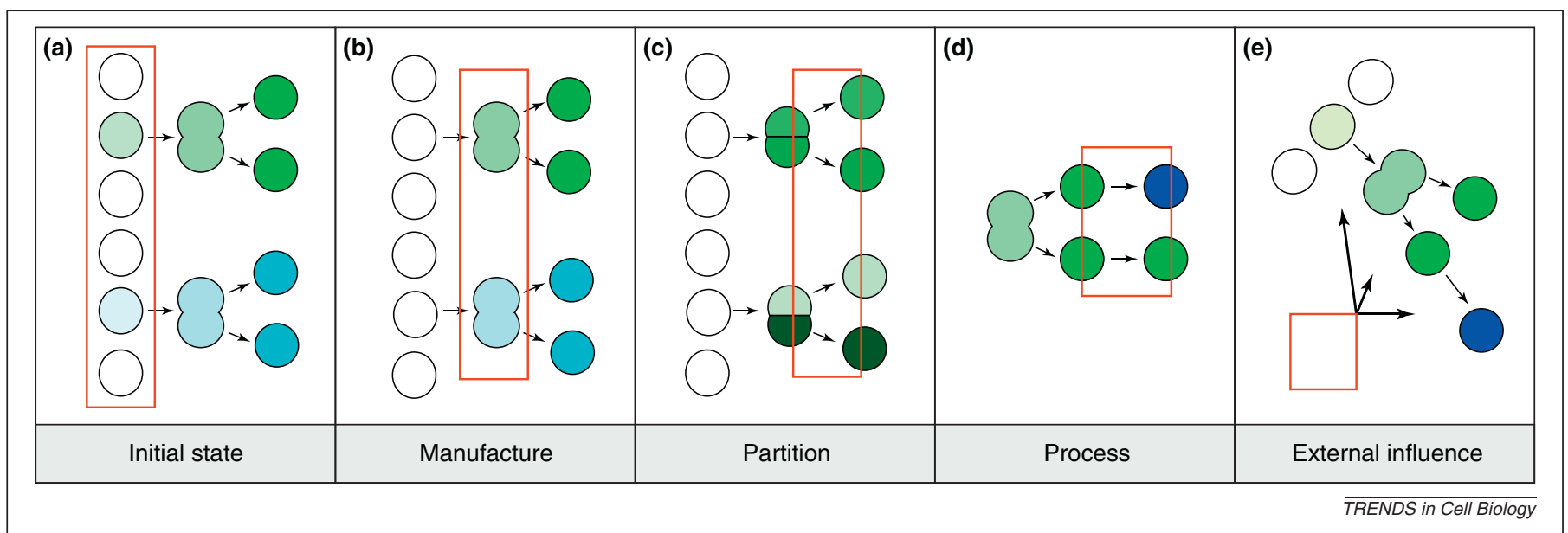

Figure 2. Sources of heterogeneity. During an adaptive immune response there are numerous potential sources of cell fate heterogeneity. These include: (a) the starting cell population may be sufficiently inhomogeneous to elicit distinct responses; (b) during mitosis, manufacture of cell components for the next generation can introduce variation; (c) during division there may be imperfect distribution of proteins between daughters (stochastic allocation or asymmetric division); (d) the machinery leading to fate may be intrinsically stochastic in its operation because it is dependent on a series of complex interactions; and (e) there may be micro-environmental differences in the signals received by each cell, resulting in distinct times to fate.

and competition - came from several distinct directions. Heterogeneity in times to divide by $\mathrm{T}$ cells was first reported by Cantrell and Smith [48] and was shown to be related to variability in receptor numbers for the growth factor IL-2 found on individual cells. More recently the introduction of division tracking by carboxyfluorescein succinimidyl ester (CFSE) intracellular labeling highlighted striking variation in the speed of division progression by similar cells arising as a consequence of a broad rightskewed distribution in times to their first division. This variability was seen for B cells as well as CD4 and CD8 T cells under many different stimulation conditions $[29,30,49-51]$. Heterogeneity in times to die was also observed for T cells and B cells placed in unstimulated culture which, similarly to times to divide, followed a broad and heavily skewed distribution $[29,30]$.

Evidence for independence of two simultaneous fates was first observed for alternative cytokine production by $\mathrm{T}$ cells [52], isotype switching decisions by B cells [53], and isotype switching and differentiation to antibody-secreting plasmablasts by B cells [41]. Time-based competition for division or death fate was first reported for $\mathrm{CD} 4^{+} \mathrm{T}$ cells in vitro [29]. Without stimulation cells died at times that were consistent with a right-skewed distribution. When a mitogenic stimulus (anti-CD3) was added an identical death curve was observed to a point, after which cell divisions, which occur at heterogeneous times, became dominant, resulting in a mix of division and death fates. A similar methodology was used later for B cells stimulated with and without the mitogenic stimulus anti-CD40 in the presence of IL-4 [30]. These data support the ACH for death or division fates leading through the first round of division. Extrapolating to further divisions formed the basis for the cyton model of competing death and division in lymphocytes $[30,54]$.

Recent data from time-lapse microscopy provide the strongest evidence for all three of these features: heterogeneity, independence and competition. Moreover, the data reveal familial properties that are not visible by other means. In particular, although stimulation of $\mathrm{B}$ cells through TLR-9 does not lead to significant isotype switching or differentiation to ASC, it has the advantage that poststimulation cells remain planar in culture and do not form 3D structures. As a result, entire family trees of all founding cells can be recorded, complete with times to each death or division.

Experiments with this system have been reported and discussed [22,55]. In these datasets population-wide heterogeneity is consistent with earlier studies, but the relatedness of family members is striking; among other deductions, these data show substantial correlations in times to death and division of collaterally consanguineous cells as well as correlations between the division times of mothers and daughters, indicating a strong founding cell influence.

Unlike stimulation of B cells through TLR-9, stimulation with anti-CD40 in the presence of IL-4 and IL-5 does lead to significant levels of isotype switching and differentiation. Under these conditions, however, cells exhibit strong homotypic adhesion and tracking entire family trees is therefore not possible. Using a combination protocol of fluorescence-activated cell sorting and filming in microwells, experiments have been reported that partially overcome these challenges, enabling the tracking of sister cells for a single generation, but not to further generations, and the recording of times to isotype switch, to differentiate to ASC, or to die or divide [33]. In those experiments, death and division are determined from bright-field images whereas isotype switching is identified through the use of anti-IgG1 bound to a fluorophore. Using B cells from a Blimp1-GFP reporter mouse, time to differentiate is identified with the time at which Blimp1, an early marker for commitment, is upregulated [56]. These data also exhibit significant sibling concordance and positive correlations in times to the same fate within siblings. This long series of observations informed the sibling-cell ACH. Extrapolations from the hypothesis ultimately proved consistent with other involved features of data from B cells stimulated with anti-CD40 [33]. 


\section{The sibling-cell $\mathrm{ACH}$}

Individual cells are subject to the intracellular $\mathrm{ACH}$, but pre-competition times to the same fate within siblings can be correlated. For example, the times to divide of siblings can be correlated and, similarly, times to die of siblings can be correlated. Within an individual cell, however, the precompetition time to die and time to divide are independent. Because same-fate times of siblings can be correlated, the impact of competition and censorship leads to a distinctive patterning of dependencies in between the times to distinct fates of siblings.

\section{Hallmarks of competition}

There are several inevitable and distinctive consequences of the ACH. We tease these out because their appearance in data provides hallmarks suggestive of the veracity of the hypothesis, whereas their absence indicates its inappropriateness. All of the following features are general properties of the ACH.

\section{Intracellular hallmarks}

Consider two possible fates within a cell, $\mathrm{X}$ and $\mathrm{Y}$, with $\mathrm{T}_{\mathrm{X}}$ being the time from birth to the occurrence of $\mathrm{X}$ and $\mathrm{T}_{\mathrm{Y}}$ the time from birth to $\mathrm{Y}$. For example, in a $\mathrm{B}$ cell $\mathrm{X}$ could be cell division and Y differentiation to ASC. Assume these fates are in competition in that if $\mathrm{X}$ occurs before $\mathrm{Y}$, then $\mathrm{Y}$ is censored and the time $\mathrm{T}_{\mathrm{Y}}$ goes unobserved, as would be the case with division and differentiation (Figure 3).

If the $\mathrm{ACH}$ is appropriate for describing $\mathrm{X}$ and $\mathrm{Y}$, then the following are inevitable consequences: (i) the postcompetition distributions of time to each fate are distinct from the underlying distributions of $\mathrm{T}_{\mathrm{X}}$ and $\mathrm{T}_{\mathrm{Y}}$, having been shaped by competition and censorship; (ii) the stiffer the competition between $\mathrm{X}$ and $\mathrm{Y}$ (i.e., the greater the overlap in their underlying distributions), the greater the censorship and thus the fewer observations of the pair of fates occurring; and, crucially, (iii) although the underlying processes are uncorrelated, there is a correlation between their observation post-competition and after censorship, and the greater the competition the greater the correlations in the observations.

Although these hallmarks are universal to all systems subject to the $\mathrm{ACH}$, a quantitative statement about them depends upon the distributions of the underlying times, $\mathrm{T}_{\mathrm{X}}$ and $\mathrm{T}_{\mathrm{Y}}$. Distinct distribution classes are likely to be appropriate for different biological processes, but a concrete illustration in a simple situation where explicit expressions are possible for all quantities of interest is provided (Box 1 and Figure 3e). The fundamental principle of competition and censorship of autonomous processes resulting in correlated times to fate when they are observed is also illustrated (Figure 3).

Returning to B cells, owing to censorship rules the order of the following events (should they be observed) must be: isotype-switching, differentiation to ASC, and then division or death. As a result, under the ACH differentiation provides greater censorship of isotype-switching than division does. Moreover, under the ACH the observed times to isotypeswitch and to differentiate to ASC should be more strongly correlated than observed times to isotype-switch and to divide. It is exactly this patterning that appears in the data contained in [33].

\section{Sibling-cell hallmarks}

Although processes leading to distinct fates within cells are stochastically independent under the $\mathrm{ACH}$, times to the same fate within siblings can be correlated. These sibling correlations also lead to distinctive features. If the samefate correlations are positive, then all of the intracellular hallmarks carry over unchanged to fates observable in distinct siblings. For example, the time to differentiate to ASC in one B cell and the time to divide of its sibling will be positively correlated.

Intercellular observations, however, allow us an additional comparison that is not possible within single cells: the time to divide of one sibling and time to die of the other sibling. If times to divide of siblings are positively correlated and the times to die of siblings are positively correlated, then on the occasions where we observe one cell divide and its sibling die, the times to these fates will also be positively correlated. The reason for this is that in one sibling division won the competition between division and death, but death won the competition in the other sibling. Because the division times of siblings are positively correlated and the death times of siblings are correlated, the only occasions when this mismatch in final fates will be observed is when death and division times are close to one another. Hence, when this mismatch is observed, the times to death and division will be positively correlated.

\section{Implications of the $\mathrm{ACH}$}

Should the ACH form an appropriate description of a given cell system, there are several deductive consequences. In general, extrapolating from an understanding of individual cells or family trees to population-level effects is challenging. In particular, given the population dynamics of the adaptive immune response forming a highly nonlinear system, prediction by intuition is not possible. From the $\mathrm{ACH}$ one can, however, extrapolate from the stochastic description of individuals to make population-level predictions that can be compared or fit with flow cytometry-style data. The mathematics necessary for this is beyond the scope of the present article, but follows the course laid down for the cyton model of intracellular competition of death and division $[30,54,57,58]$ while employing multitype branching processes.

This mathematical method records the number of cells of each type in each generation at any given time. The methodology allows the evaluation of the mean number of cells per fate per generation as well as variances and covariances in numbers of cells of distinct type as a result of the intrinsic variability in the cell populationsize dynamics. Other quantities of interest, such as the probability that cells of a given type are not present, are readily calculable. For biologically reasonable distribution classes for times to fate, such as the log-normal, Weibull and other right-skewed distributions, this approach does not give closed-form expressions for these quantities, but is accommodated by straightforward numerics. 


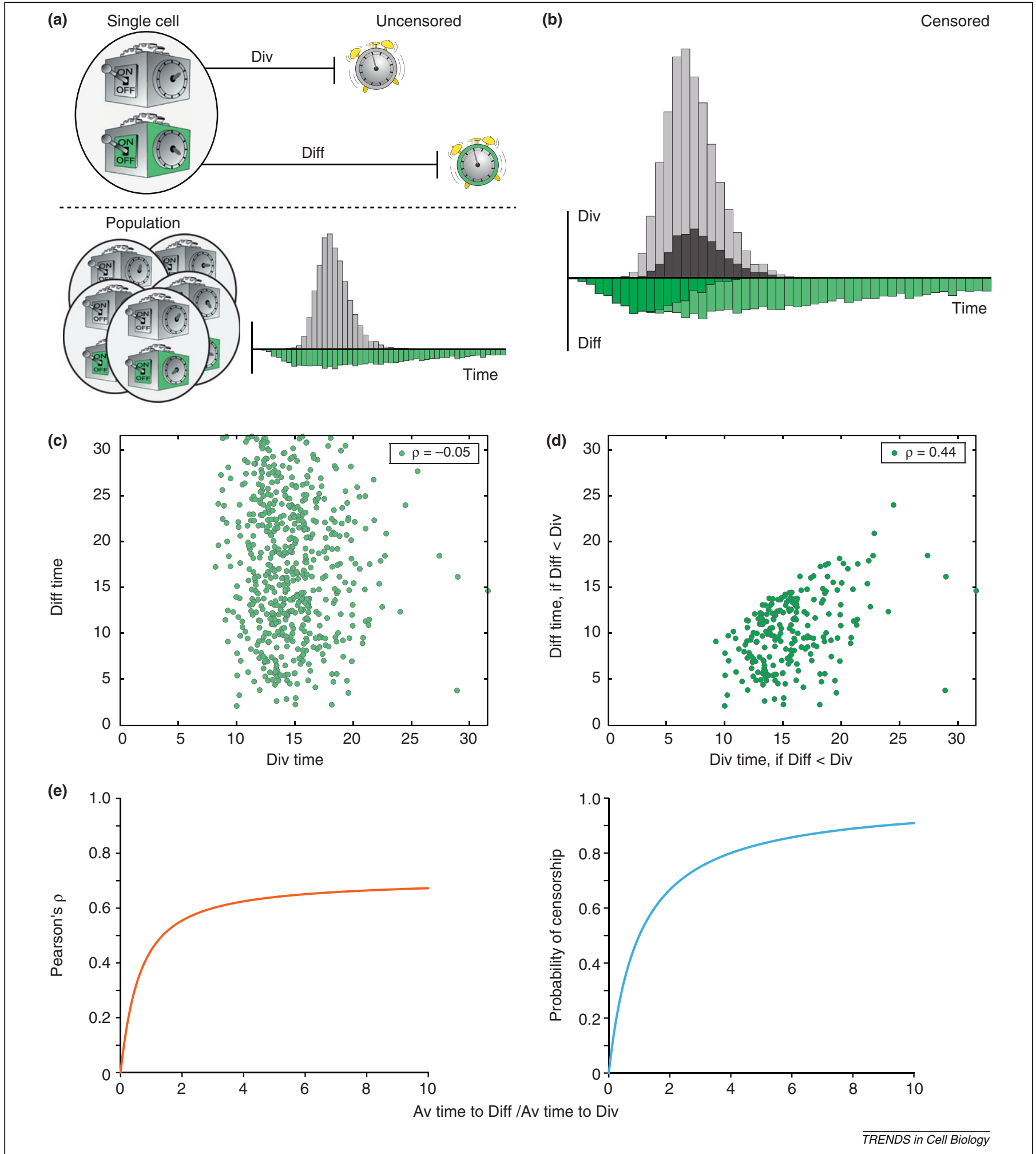

Figure 3. Hallmarks of competition. The autonomous competition hypothesis (ACH) posits that multiple fates can be set in competition within an individual cell. As a consequence distinctive features will arise in the population due to the influence of a winning fate over the course of other competing fates. (a) In the upper panel a cell picks times to two distinct fates - division (div) and development to an antibody-secreting cell (ASC) - from distinct distributions. In this example the cell divides but does not differentiate (diff). The lower panel illustrates histograms of times chosen by a collection of cells, with division time being plotted above differentiation time. Panel (b) shows the consequence of competition and censorship. For cells that differentiate and go on to divide, the darkened histograms illustrate the distributions of these times. Late differentiation times are more likely to be censored, whereas early division times are more likely to cause censorship. Panel (c) shows a scatter plot of simulated precompetition times to differentiate and divide. Because each process is stochastically independent, the empirical correlation coefficient of the pre-competition data is -0.05 Panel (d) shows the impact of competition, where all data above the $45^{\circ}$ line have been censored. Despite the independence of the underlying processes, competition has induced a correlation coefficient of 0.44 in the observed data. Such correlations are a hallmark of autonomous competing processes. (e) If times to differentiate and times to divide are exponentially distributed, Pearson's $\rho$ (left panel) and the probability of censorship (right panel) for time to differentiate are explicitly calculable as a function of the average time to differentiate divided by the average time to divide (Box 1), which are plotted here. The greater the competition, the greater the correlation in observed times to fate and the greater the likelihood of censorship. 


\section{Box 1. Hallmarks of competition}

As an illustration of the three hallmarks of intracellular competition, consider the hypothetical case of two fates $X$ and $Y$ corresponding to division and differentiation to ASC of a B cell. For computational tractability, assume that the times from birth to these fates, $T_{X}$ and $T_{Y}$, are exponentially distributed with rates $\mu$ and $\lambda$ respectively so that their probability density functions are

$f_{X}(t)=\mu \exp (-\mu t)$ and $f_{Y}(t)=\lambda \exp (-\lambda t)$.

Elementary calculations reveal the following, which are illustrated graphically in Figure $3 \mathrm{e}$.

(i) The distribution of post-competition, censored values of times to differentiate remains exponential, but now with rate

$\lambda+\mu, \quad f_{Y \mid Y<X}(t)=(\lambda+\mu) \exp (-(\lambda+\mu) t)$.

That is, given one observes a cell differentiate and then divide, the observed time to differentiate distribution is more focused on smaller times than the pre-competition distribution. This occurs because if the pre-competition value was large, division would be likely to win the competition and so it would go unobserved.

The post-competition distribution of time to divide when observed in combination with differentiation is also reshaped. It is no longer exponential, but instead has the form

$\mathrm{f}_{\mathrm{X} \mid \mathrm{Y}<\mathrm{X}}(\mathrm{t})=(\lambda+\mu) \mu / \lambda(\exp (-\mu \mathrm{t})-\exp (-(\lambda+\mu) \mathrm{t})$

giving a greater likelihood of seeing larger values than in the precompetition distribution. This occurs because if the pre-competition time to divide was small it is unlikely that differentiation could occur before it, and thus it would be censored.

(ii) We can quantify the likelihood that a sample will be censored. With $r=\mu / \lambda$ being the pre-competition average time to differentiation divided by the average time to division, the probability that censorship occurs is $r /(1+r)$. When differentiation typically occurs much earlier than division, $r$ is small and there is little censorship. When time to differentiate typically takes values greater than time to division, $r$ is large and there is substantial censorship.

(iii) We can also quantify the correlation in the observed times to fate induced by competition and censorship. Pearson's $\rho$ for the pre-competition $T_{X}$ and $T_{Y}$ is zero due to independence, but Pearson's $\rho$ for the observed times, $T_{X}$ and $T_{Y}$, given that $T_{Y}<$ $T_{X}$, is

$\rho=r /\left(r^{2}+(1+r)^{2}\right)^{0.5}$

which is positive. When differentiation occurs much earlier than division on average, it tends to zero, but when differentiation occurs much later than division on average it tends to $(1 / 2)^{0.5} \approx$ 0.707 (Figure 3e)

Through using the ACH and extrapolations from it to analyze data, one can isolate the effects of genetic changes or drugs on the autonomous modules of cells. This allows calculation of population effects when found in combination. Indeed, if a desirable population-level outcome is known, the mathematics can propose promising combination therapies for further intervening and creating the desired outcome, whether it is suppressive or stimulatory.

\section{The broader significance of the ACH}

The data from recent studies of the adaptive immune response reveal a more complex and interesting patterning of cell fate than would perhaps have been predicted, with substantial heterogeneity and complex correlations in times to fate. At a single-cell level, they are consistent with the first-order approximation that autonomous stochastic processes, timed from mitosis or an activating trigger, are underway within each cell leading to each potential fate, with competition between these processes determining the fate of a cell. The hallmarks of this hypothesis are distinctive and hard to mistake, and thus serve as a stern test of its appropriateness.

Studies with lymphocytes raise the question of how ubiquitous this system might be and where else hallmarks of competition might be found. Intriguingly, a recent paper [32] reported that a competing molecular race, consistent with intracellular $\mathrm{ACH}$, is responsible for the allocation of a proportion of the population to either competence or spore formation within Bacillus subtilis, suggesting the strategy of temporal competition may be an ancient one. Within mammals, single-cell tracking has been developed to trace hematopoietic stem cells $[59,60]$, retinal progenitor cells [61], intestinal crypt cells [62] and oligodendrocytes [63], and each could be studied to test for features of the ACH.

Some of these other cell systems have an advantage over $\mathrm{B}$ and $\mathrm{T}$ lymphocytes in that they can produce complete family trees. Differentiating B cells express adhesion molecules, making tracking beyond a single generation a serious challenge. To collect family-tree data for differentiating lymphocytes new methods will have to be developed to prevent aggregation and loss of individual cell identity. This could be by anchoring or isolating cells or by developing suitable microfluidic devices $[64,65]$. Differentiating T cells exhibit additional challenges because they may adopt a fate, such as the ability to produce a particular cytokine, whose presence can only be determined by interrogation. As a consequence, the time at which the fate is adopted may not be observed leading to the question of how can one reliably test for the appropriateness of the $\mathrm{ACH}$ in that case.

B cells stimulated to proliferate, but not to differentiate, indicate there is more heterogeneity between distinct family trees than there is within them. This suggests that mathematical models treat the family tree as the fundamental unit of heterogeneity instead of individual cells. Data describing entire family trees, as may be possible with some of the other cell types mentioned above, could be used to inform a family-tree version of the hypothesis, extending the sibling-cell ACH. Such data could also offer insight into the relative strength of contribution of the potential sources of randomness and heterogeneity described in Figure 2. Understanding the significance of each component could have a substantial bearing on determining a strategy for manipulation of the resulting population dynamics.

If in a given cell system the hallmarks of the ACH are not found, then even this is informative because it indicates that the processes governing fate determination are not autonomous and may be subject to joint control. In particular, it suggests investigating the possibility and identification of shared componentry within the cell resulting in the coupling of fates. Such a system may exhibit hallmarks of an alternative hypothesis (Box 2).

Should its hallmarks be present, the $\mathrm{ACH}$ provides a simple explanation for highly complex and apparently daunting data, although many outstanding questions remain (Box 3). In general, the introduction of each additional fate doubles the number of potential cell types, leading to exponential growth in the complexity of the data. With the $\mathrm{ACH}$, however, an additional fate only requires the 


\section{Box 2. Hallmarks of alternative hypotheses}

(i) Determinism

From this viewpoint, two identical cells that are exposed to the same stimulus will lead to two identical copies of the one family tree. For this to be consistent with reports of substantial heterogeneity in fates and times to them, there must be subtle, but significant, differences in the initial cells or in micro-environmental nature of the stimulus (see Figure 2 for a summary of the sources of heterogeneity for a developing lineage tree).

(ii) Fate selection at birth

An alternate stochastic hypothesis posits that cells make their fate decisions at birth followed by choosing a time to those fates based on a stochastic fate-dependent distribution. If the decision to pursue each fate is made independently, then the population-level number of cells exhibiting each fate must be consistent with a product distribution. For example, imagine that a cell can take on two fates, $\mathrm{F}_{1}$ and $\mathrm{F}_{2}$, and that these occur with probabilities $P_{1}$ and $P_{2}$; the proportion of cells that exhibit both fates would then be $P_{1} \cdot P_{2}$. This hypothesis is strongly rejected by the data in [33], suggesting that if fate selection mimics this paradigm, each fate choice cannot be made independently. A second complication necessary to be consistent with experiments is that, once selected, the time to each fate combination is heavily dependent on the combination, necessitating model parameterization that grows exponentially with the number of fates.

characterization of one new autonomous process, greatly enhancing one's ability to interpret these data. If it is appropriate, the ACH offers a methodology for understanding the impact of manipulation through the application of drugs at the level of individual cells and the extrapolation of its consequences to the population-level

\section{Box 3. Outstanding questions}

- All of the evidence for $\mathrm{ACH}$ in the literature is based on in vitro imaging and in vitro cultures; can an in vivo system be developed where the $\mathrm{ACH}$ can be tested in situ?

- There are many potential sources of heterogeneity and randomness in growing, differentiating cell systems. Can systems be developed to isolate and measure the relative importance of each potential component?

- B cell fates such as isotype switching, differentiation to ASC, division or death are all recognizable and apparent. For other cell systems, this is not always true. For example, $\mathrm{T}$ cells can differentiate and be capable of cytokine creation, but must be interrogated to discover this. How can one convincingly check for the hallmarks of the $\mathrm{ACH}$ in this setting where times are not directly observable?

- Lymphoblasts and plasmablasts exhibit strong homotypic adhesion, making the observations of entire family trees impossible as it stands. Can an experimental system based on anchoring cells or the use of microfluidic devices be developed to overcome this drawback?

- For cell systems where entire family trees can be observed, what is the appropriate family-tree level version of the $\mathrm{ACH}$ ?

- The $\mathrm{ACH}$ categorizes cells into either possessing or not possessing a fate. For some fates, such as death or division, this is reasonable. For other fates, however, a quantitative level, such as the number of receptors generated that are associated with the fate, may be functionally necessary information. How should these subtleties be incorporated?

- Having identified cytokines whose quantitative influence on death and division is understood, can one predict the population-level consequence of continuous changes in cytokine concentration over time? dynamical response, making complex cell systems amenable to quantitative prediction.

\section{Acknowledgments}

We thank the inimitable Jie H.S. Zhou (Walter and Eliza Hall Institute) for assistance in the production of figures and David Sandström (NorBAC) for inspiration. This work was supported by the Australian National Health and Medical Research Council (program grant 575500) and Victorian State Government operational infrastructure support.

\section{References}

1 Sallusto, F. et al. (2004) Central memory and effector memory T cell subsets: function, generation, and maintenance. Annu. Rev. Immunol. 22, 745-763

2 MacLennan, I.C. et al. (2003) Extrafollicular antibody responses. Immunol. Rev. 194, 8-18

3 Jenkins, M.R. et al. (2007) Heterogeneity of effector phenotype for acute phase and memory influenza A virus-specific CTL. J. Immunol. 179, 64-70

4 Seder, R.A. et al. (2008) T-cell quality in memory and protection: implications for vaccine design. Nat. Rev. Immunol. 8, 247-258

5 Goodnow, C.C. et al. (2010) Control systems and decision making for antibody production. Nat. Immunol. 11, 681-688

6 Garcia, S. et al. (1999) Following the development of a CD4 T cell response in vivo: from activation to memory formation. Immunity 11, 163-171

7 Jenkins, M.K. et al. (2001) In vivo activation of antigen-specific CD4 T cells. Annu. Rev. Immunol. 19, 23-45

8 De Boer, R.J. et al. (2003) Different dynamics of CD4 ${ }^{+}$and $\mathrm{CD}^{+} \mathrm{T}$ cell responses during and after acute lymphocytic choriomeningitis virus infection. J. Immunol. 171, 3928-3935

9 Strasser, A. and Pellegrini, M. (2004) T-lymphocyte death during shutdown of an immune response. Trends Immunol. 25, 610-615

10 Williams, M.A. et al. (2008) Rapid culling of the $\mathrm{CD}^{+} \mathrm{T}$ cell repertoire in the transition from effector to memory. Immunity 28, 533-545

11 Kalia, V. et al. (2006) Differentiation of memory B and T cells. Curr. Opin. Immunol. 18, 255-264

12 Oracki, S.A. et al. (2010) Plasma cell development and survival. Immunol. Rev. 237, 140-159

13 Stavnezer, J. et al. (2008) Mechanism and regulation of class switch recombination. Annu. Rev. Immunol. 26, 261-292

14 Seder, R.A. and Ahmed, R. (2003) Similarities and differences in CD4 ${ }^{+}$ and $\mathrm{CD}^{+}$effector and memory $\mathrm{T}$ cell generation. Nat. Immunol. 4, 835-842

15 Kaech, S.M. and Wherry, E.J. (2007) Heterogeneity and cell-fate decisions in effector and memory CD8+ T cell differentiation during viral infection. Immunity 27, 393-405

16 Zhu, J. and Paul, W.E. (2010) Peripheral CD4 ${ }^{+}$T-cell differentiation regulated by networks of cytokines and transcription factors. Immunol. Rev. 238, 247-262

17 Stemberger, C. et al. (2007) A single naive $\mathrm{CD}^{+} \mathrm{T}$ cell precursor can develop into diverse effector and memory subsets. Immunity 27, 985-997

18 Schepers, K. et al. (2008) Dissecting T cell lineage relationships by cellular barcoding. J. Exp. Med. 205, 2309-2318

19 Gerlach, C. et al. (2010) One naive T cell, multiple fates in $\mathrm{CD}^{+} \mathrm{T}$ cell differentiation. J. Exp. Med. 207, 1235-1246

20 Rieger, M.A. et al. (2009) Hematopoietic cytokines can instruct lineage choice. Science 325, 217-218

21 Rieger, M.A. and Schroeder, T. (2009) Analyzing cell fate control by cytokines through continuous single cell biochemistry. J. Cell. Biochem. 108, 343-352

22 Hawkins, E.D. et al. (2009) A single-cell pedigree analysis of alternative stochastic lymphocyte fates. Proc. Natl. Acad. Sci. U.S.A. 106, 13457-13462

23 Day, D. et al. (2009) A method for prolonged imaging of motile lymphocytes. Immunol. Cell Biol. 87, 154-158

24 Jenkins, M.R. et al. (2009) Visualizing CTL activity for different CD8 ${ }^{+}$ effector T cells supports the idea that lower TCR/epitope avidity may be advantageous for target cell killing. Cell Death Differ. 16, 537-542

25 Chang, J.T. et al. (2011) Asymmetric proteasome segregation as a mechanism for unequal partitioning of the transcription factor T-bet during T lymphocyte division. Immunity 34, 492-504 
26 Oliaro, J. et al. (2010) Asymmetric cell division of T cells upon antigen presentation uses multiple conserved mechanisms. J. Immunol. 185 , 367-375

27 Mittelbrunn, M. et al. (2009) Imaging of plasmacytoid dendritic cell interactions with T cells. Blood 113, 75-84

28 Guldevall, K. et al. (2010) Imaging immune surveillance of individual natural killer cells confined in microwell arrays. PLoS ONE 5, e15453

29 Gett, A.V. and Hodgkin, P.D. (2000) A cellular calculus for signal integration by T cells. Nat. Immunol. 1, 239-244

30 Hawkins, E.D. et al. (2007) A model of immune regulation as a consequence of randomized lymphocyte division and death times. Proc. Natl. Acad. Sci. U.S.A. 104, 5032-5037

31 Huang, H.C. et al. (2010) Stochastic competition between mechanistically independent slippage and death pathways determines cell fate during mitotic arrest. PLoS ONE 5, e15724

32 Kuchina, A. et al. (2011) Temporal competition between differentiation programs determines cell fate choice. Mol. Syst. Biol. 7, 557

33 Duffy, K.R. et al. (2012) Activation-induced B cell fates are selected by intracellular stochastic competition. Science 335, 338-341

34 Kelso, A. (1999) Educating T cells: early events in the differentiation and commitment of cytokine-producing $\mathrm{CD}^{+}$and $\mathrm{CD}^{+} \mathrm{T}$ cells. Springer Semin. Immunopathol. 21, 231-248

35 Murphy, K.M. et al. (2000) Signaling and transcription in T helper development. Annu. Rev. Immunol. 18, 451-494

36 Guo, L. et al. (2005) Probabilistic regulation of IL-4 production. J. Clin. Immunol. 25, 573-581

37 Hodgkin, P.D. et al. (1996) B cell differentiation and isotype switching is related to division cycle number. J. Exp. Med. 184, 277-281

38 Gett, A.V. and Hodgkin, P.D. (1998) Cell division regulates the T cell cytokine repertoire, revealing a mechanism underlying immune class regulation. Proc. Natl. Acad. Sci. U.S.A. 95, 9488-9493

39 Bird, J.J. et al. (1998) Helper T cell differentiation is controlled by the cell cycle. Immunity 9, 229-237

40 Tangye, S.G. et al. (2003) A division-linked mechanism for the rapid generation of Ig-secreting cells from human memory B cells. $J$. Immunol. 170, 261-269

41 Hasbold, J. et al. (2004) Evidence from the generation of immunoglobulin G-secreting cells that stochastic mechanisms regulate lymphocyte differentiation. Nat. Immunol. 5, 55-63

42 Tangye, S.G. and Hodgkin, P.D. (2004) Divide and conquer: the importance of cell division in regulating B-cell responses. Immunology 112, 509-520

43 Jenkins, M.R. et al. (2008) Cell cycle-related acquisition of cytotoxic mediators defines the progressive differentiation to effector status for virus-specific $\mathrm{CD}^{+}{ }^{+} \mathrm{T}$ cells. J. Immunol. 181, 3818-3822

$44 \mathrm{Nolz}$, J.C. et al. (2012) Division-linked generation of deathintermediates regulates the numerical stability of memory CD8 T cells. Proc. Natl. Acad. Sci. U.S.A. 109, 6199-6204

45 Yates, A. et al. (2007) Reconstruction of cell population dynamics using CFSE. BMC Bioinform. 8, 196

46 Zilman, A. et al. (2010) Stochastic models of lymphocyte proliferation and death. PLoS ONE 5, e12775
47 Nordon, R.E. et al. (2011) Multi-type branching models to describe cell differentiation programs. J. Theor. Biol. 277, 7-18

48 Cantrell, D.A. and Smith, K.A. (1984) The interleukin-2 T-cell system: a new cell growth model. Science $224,1312-1316$

49 Deenick, E.K. et al. (2003) Stochastic model of T cell proliferation: a calculus revealing IL-2 regulation of precursor frequencies, cell cycle time, and survival. J. Immunol. 170, 4963-4972

50 Hommel, M. and Hodgkin, P.D. (2007) TCR affinity promotes $\mathrm{CD} 8^{+} \mathrm{T}$ cell expansion by regulating survival. J. Immunol. 179, 2250-2260

51 Tangye, S.G. et al. (2003) Intrinsic differences in the proliferation of naive and memory human $\mathrm{B}$ cells as a mechanism for enhanced secondary immune responses. J. Immunol. 170, 686-694

52 Kelso, A. and Gough, N.M. (1988) Coexpression of granulocytemacrophage colony-stimulating factor, gamma interferon, and interleukins 3 and 4 is random in murine alloreactive T-lymphocyte clones. Proc. Natl. Acad. Sci. U.S.A. 85, 9189-9193

53 Deenick, E.K. et al. (1999) Switching to IgG3, IgG2b, and IgA is division linked and independent, revealing a stochastic framework for describing differentiation. J. Immunol. 163, 4707-4714

54 Subramanian, V.G. et al. (2008) Determining the expected variability of immune responses using the cyton model. J. Math. Biol. 56, 861-892

55 Markham, J.F. et al. (2010) A minimum of two distinct heritable factors are required to explain correlation structures in proliferating lymphocytes. J. R. Soc. Interface 7, 1049-1059

56 Kallies, A. et al. (2004) Plasma cell ontogeny defined by quantitative changes in blimp-1 expression. J. Exp. Med. 200, 967-977

57 Duffy, K.R. and Subramanian, V.G. (2009) On the impact of correlation between collaterally consanguineous cells on lymphocyte population dynamics. J. Math. Biol. 59, 255-285

58 Wellard, C. et al. (2010) The effect of correlations on the population dynamics of lymphocytes. J. Theor. Biol. 264, 443-449

59 Rieger, M.A. et al. (2009) Improved prospective identification of megakaryocyte-erythrocyte progenitor cells. Br. J. Haematol. 144, 448-451

60 Rieger, M.A. and Schroeder, T. (2008) Exploring hematopoiesis at single cell resolution. Cells Tissues Organs 188, 139-149

61 Gomes, F.L. et al. (2011) Reconstruction of rat retinal progenitor cell lineages in vitro reveals a surprising degree of stochasticity in cell fate decisions. Development 138, 227-235

62 Snippert, H.J. et al. (2010) Intestinal crypt homeostasis results from neutral competition between symmetrically dividing Lgr5 stem cells. Cell 143, 134-144

63 Hyrien, O. et al. (2006) Stochastic modeling of oligodendrocyte generation in cell culture: model validation with time-lapse data Theor. Biol. Med. Model. 3, 21

64 Rowat, A.C. et al. (2009) Tracking lineages of single cells in lines using a microfluidic device. Proc. Natl. Acad. Sci. U.S.A. 106, 18149-18154

65 Falconnet, D. et al. (2011) High-throughput tracking of single yeast cells in a microfluidic imaging matrix. Lab Chip 11, 466-473 